\title{
Impact of Supporting People with Advanced Parkinson's Disease on Carer's Quality of Life and Burden
}

This article was published in the following Dove Press journal:

Neuropsychiatric Disease and Treatment

\author{
Nicola Modugno, (iD) \\ Angelo Antonini, (iD) ${ }^{2}$ \\ Alessandro Tessitore, ${ }^{3}$ \\ Pietro Marano, 4 \\ Francesco Ernesto Pontieri, 5,6 \\ Nicola Tambasco, iD ${ }^{7}$ \\ Margherita Canesi, ${ }^{8,9}$ \\ Giovanni Fabbrini, (D) ${ }^{10,11}$ \\ Mariachiara Sensi, ${ }^{12}$ \\ Rocco Quatrale, ${ }^{13}$ Paolo Solla, ${ }^{14}$ \\ Giovanni Defazio, ${ }^{14}$ Gabriella Melzi, ${ }^{15}$ \\ Giuliana Gualberti, (DD ${ }^{15}$ \\ Leonardo Lopiano (iD) ${ }^{16}$
}

'Neurology Unit, IRCCS Neuromed, Pozzilli, Italy ${ }^{2}$ Parkinson and Movement Disorder Unit.

Department of Neuroscience, University of Padua,

Padua, Italy; ${ }^{3}$ Department of Medical and Surgery

Sciences, University of Campania, "Luigi Vanvitelli",

Naples, Italy; ${ }^{4}$ Casa di Cura Madonna del Rosario,

Raggruppamento di Riabilitazione, Catania, Italy;

${ }^{5}$ Department NESMOS, "Sapienza" University,

Sant'Andrea Hospital, Roma, Italy; ${ }^{6}$ IRCCS

Fondazione Santa Lucia, Roma, Italy; ${ }^{7}$ Department

of Neurology, Perugia General Hospital and

University of Perugia, Perugia, Italy; ${ }^{8}$ Dipartimento

di Riabilitazione Malattia di Parkinson e Disordini

del Movimento, Gravi Cerebrolesioni Acquisite,

Italia Hospital - Ospedale "Moriggia-Pelascini”,

Gravedona ed Uniti, Como, Italy; ${ }^{9}$ Centro

Parkinson e Disordini del Movimento, CTO, G Pini,

Milano, Italy; ${ }^{10}$ Department of Human

Neurosciences, Sapienza University of Rome,

Rome, Italy; "IRCCS Neuromed, Pozzilli, Isernia,

Italy; ${ }^{12}$ Neurology Unit, Hospital Sant'Anna,

Ferrara, Italy; ${ }^{13}$ Neurology Unit, Hospital dell'Angelo, Mestre, VE, Italy; ${ }^{14}$ Neurology Unit,

Policlinico Universitario Monserrato, Cagliari, Italy,

${ }^{15}$ Medical Department, AbbVie Srl, Roma, Italy;

${ }^{16}$ Department of Neuroscience "Rita Levi

Montalcini" University of Torino, Azienda

Ospedaliero-Universitaria Città Della Salute e

Della Scienza di Torino, Torino, Italy

Correspondence: Gabriella Melzi

AbbVie Srl, Viale dell'Arte 25, Roma 00I44, Italy Tel +3906928924346

Email gabriella.melzi@abbvie.com
Purpose: The aim of this study was to assess the burden and the quality of life (QoL) perceived by caregivers assisting advanced Parkinson's disease (PD) patients.

Patients and Methods: Consecutive advanced PD patients treated with levodopa/carbidopa intestinal gel (LCIG) or continuous subcutaneous apomorphine infusion (CSAI) or care as usual (CU) and their care partners were recruited during routine visits according to a cross-sectional design. Caregiver's distress was assessed by Zarit Burden Interview (ZBI) and a QoL survey to evaluate and understand the burden experienced by care partners during family and working activities.

Results: A total of 126 patients (53 LCIG, 19 CSAI and $54 \mathrm{CU}$ ) and their care partners were enrolled. The ZBI score boxplot showed that LCIG and CU populations have a similar distribution (ZBI inter-quartile range [IQR] values respectively 18-42 for LCIG and 19-43 for CU group), while the CSAI group has a wider score range (IQR 16-52). Caregivers assisting patients in treatment with LCIG have more time to perform family or household duties $(p=0.0022)$, or to engage in leisure activities $(p=0.0073)$ compared to $C U$, while no difference was found when compared to CSAI group. Approximately 50\% of the care partners showed mood changes in the last 6 months and LCIG and CSAI had less impact on caregiver's mood compared to CU. Patients treated with LCIG were more independent in taking a bath or shower without assistance and were more able to move and walk without assistance.

Conclusion: Care partners of advanced PD patients treated with device-aided therapies have more time for their own life and a better perception of their QoL with a tendency to an improvement of mood compared with those of patients treated with $\mathrm{CU}$.

Keywords: advanced Parkinson's disease, levodopa/carbidopa, intestinal infusion, caregiver burden, quality of life; QoL

\section{Introduction}

Parkinson's disease (PD) is a neurodegenerative disorder characterized by progressively disabling motor and non-motor symptoms (NMS), progressive loss of patient's autonomy, deterioration of functional and psychosocial patient's condition, and worsening of Quality of Life (QoL). ${ }^{1,2}$

The progressive course of the disease causes needs of assistance for daily activities and has a negative impact not only on patients but also on their caregivers. ${ }^{3}$ Recent studies suggest that the progressive disability, patient's mood, speech difficulties, and cognitive deterioration are the main factors contributing to caregiver burden and poorer caregiver's QoL. ${ }^{4-9}$ Most PD patients 
are cared for in their own homes and spouses are the main informal caregivers. ${ }^{10,11}$

During the disease progression, the caregiver of a PD patient assumes many responsibilities, including medication administration, care coordination, prompting for selfcare, surveillance of falls, provisions of emotional support. ${ }^{3}$ Having a caregiver in a good health has been associated with better outcomes in morbidity, mortality, and patient's QoL. ${ }^{12}$ Conversely, the caregiver's burden and its mainly associated symptoms (fatigue, sleep disorders, hypertension), correlate with the illness of the respective PD patients. ${ }^{13,14}$

The advanced stage of PD is also characterized by a worsening in QoL and caregiver burden. ${ }^{15}$ At this stage, the therapeutic options include device-aided interventions, such as deep brain stimulation (DBS), continuous subcutaneous apomorphine infusion (CSAI), and levodopa/carbidopa continuous intestinal infusion gel (LCIG) or oral therapy.

Few evidence have linked caregiver burden to the treatment of advanced PD patients and these studies are mainly referred to STN-DBS or LCIG. ${ }^{3,16-18,}$ Despite the effectiveness of STN-DBS in the treatment of motor symptoms and its benefit for patient-rated QoL have been widely demonstrated, its impact on caregiver burden is less clear. ${ }^{19}$ Recent evidence reported that over $50 \%$ of the caregivers rated their well-being as negative after 1 year of STN-DBS surgery, despite the positive patientrated outcomes. ${ }^{16}$ In addition, a recent longitudinal study has reported that caregivers who showed high levels of burden at baseline, as well as caregivers who referred high impulsivity and poor empathy in their partner with PD, generally maintained the same profile during the longitudinal follow-up. ${ }^{19}$ According to other Authors the possible conclusion is that STN-DBS does not change caregiver burden. ${ }^{19,20}$ This evidence was in line with the conclusions of another recent trial showing that treatment with DBS was not associated with a lower caregiver burden compared to treatment with other antiparkinsonian therapies. ${ }^{21}$

Moreover, few data on the effect of apomorphine on caregiver burden are available. ${ }^{18}$ In our previous report of the results from the PREDICT study, whose aim was to describe the burden of carers of advanced PD patients either treated with CSAI, LCIG, or continuing with their $\mathrm{CU}$, we showed that caregiver burden tended to be lower when patients were treated with LCIG than with $\mathrm{CU}{ }^{18}$

In this further analysis of the same study, our aim was to understand if reduced burden could have an impact on caregiver's QoL and their ability to perform their daily activities. We therefore describe more in detail an evaluation of caregiver burden due to the daily assistance to the patient and his/her QoL perception as assessed by a custom-made questionnaire during the study.

\section{Patients and Methods}

\section{Study Design}

The PREDICT observational study was conducted at 13 Movement Disorder (MD) Centers in Italy, according to a cross-sectional design, in a series of consecutive advanced PD patients and their caregivers recruited during routine follow-up visits planned at the single centers.

\section{Patient and Caregiver Selection}

Advanced PD patients already in treatment with optimized CSAI, LCIG, or CU since at least 6 months up to 3 years were enrolled in this study. The inclusion criteria to verify the advanced stage included the presence of at least 3 hours OFF period per day or $>25 \%$ of daily time spent in OFF as assessed by United Parkinson's Disease Rating Scale (UPDRS)-IV item 39. Further inclusion criteria were the presence of a family adult carer who had provided regular daily assistance $(\geq 3$ hours per day) to the patient for at least 6 months.

The history or presence of any severe condition that might interfere with caregiver burden assessments, previous treatment with LCIG, CSAI, or DBS, mild to severe cognitive dysfunction/dementia (i.e., Mini-Mental State Examination score $<24$ or per clinical judgment), and Hoehn \& Yahr (H\&Y) stage 5 in OFF condition in the previous 12 months were considered as exclusion criteria.

The present study was approved by the Ethics Committee of each local health authority (Supplementary Table 1) and each patient had to be able to understand and provide their informed consent as well as their care partners provided informed consent. Patients with mild to severe cognitive dysfunction/dementia, were excluded in order not to interfere with the capacity consent. The study was conducted according to the International Conference on Harmonization Good Clinical Practices.

\section{Assessments}

The demographic and clinical characteristics (including UPDRS-IV Item 39 and H\&Y assessment) of the populations were described in the previous paper, as well as the mean Zarit Burden Interview (ZBI) score and related factors. ${ }^{18}$ The ZBI assesses the impact of the disease on 
caregiver's emotional, physical, social, and financial wellbeing $^{22}$ using a 22 -item questionnaire with a 5 -point scale, from $0=$ never to $4=$ nearly always. ${ }^{11}$ The ZBI total score is categorized as follows: 0 to 20 (little or no burden), 21 to 40 (mild to moderate burden), 41 to 60 (moderate to severe burden), and 61 to 88 (severe burden). Scores were further aggregated into 2 categories: ZBI total score from 0 to 40 (little to moderate burden) and from 41 to 88 (moderate to severe burden).

Patients treated with LCIG or CSAI and their caregivers were asked to self-assess their current QoL compared with the previous $\mathrm{CU}$ according to a score rated from 0 (very negative) to 10 (very positive); patients' QoL was assessed using the 8-item Parkinson's Disease Questionnaire (PDQ-8). ${ }^{23}$

Both ZBI and PDQ-8 were provided in their Italian validated version according to copyright holders' instructions. Caregivers were asked to complete a dedicated custom-made questionnaire whose aim was to estimate the degree of involvement of familial assistance, working capacity, and working habits changes. This questionnaire has been developed as a simplified version of the Scale of Quality of Life of Care-Givers (SQLC, Glozman et al), ${ }^{24}$ adapted to create a short version for an easy assessment of the QoL of care partners. The complete QoL questionnaire is reported in the result section; it consisted of 7 questions on the caregivers' ability to perform daily activities related to personal or family needs and on their opinion about the real need of help and assistance of the relative patients in daily activities. Caregivers were asked to give their opinion on their perception since the patient was optimized with the current therapy, answering to each question with one the following scores: $0=$ yes, more than before; $1=$ yes, as before; 2 = yes, but with some difficulties compared to before; $4=$ no. Furthermore, caregivers were also asked whether in the last 6 months they complained of one or more of the following: mood changes (worsening or improvement), depressive state, body weight reduction, sleep disturbances or insomnia, anxiety.

Physicians provided paper questionnaires to caregivers in an envelope and invited them to complete them in the outpatient room.

\section{Statistical Analysis}

A sample size of 120 caregivers/subjects (according to a 1:2 ratio, CU:CSAI/LCIG) was calculated to estimate a statistically significant difference in the mean ZBI score equal or superior to 13 points, as previously described. ${ }^{18}$
The per-protocol population (PP) was defined as all enrolled subjects without a main protocol violation. All statistical tables, figures, and analyses were produced using SAS $^{\circledR}$ for Windows release 9.4 (64-bit) or later (SAS Institute Inc., Cary, NC, USA). Patient and caregiver profile data were analyzed only in the PP population by means of descriptive statistics. The categorical variables were assessed by Chi square or Fisher exact test, as appropriate, whilst the continue variables by $t$-test. Whenever necessary, normality was assessed by means of the Shapiro-Wilk test and with graphical methods. In case of non-normality, a nonparametric test/model was adopted. A two-sided $p<0.05$ was considered statistically significant. In the case of comparisons between LCIG treatment versus CSAI and CU treatment, the significance level was set at 0.025 .

According to copyright holder instructions ZBI total score was calculated only for questionnaires with $\geq 18$ responses out of 22 questions. In case of $\leq 4$ missing questions, the average scores from valid responses were rounded to the nearest integer and used to complete any missing fields.

ZBI single questions data have been also aggregated as "never+rarely" vs. "Sometimes+Quite frequently+nearly always" in the comparison of LCIG vs. CSAI and CU responses.

\section{Results}

The enrollment of patients and their caregivers was performed between September 2014 and September 2015. Out of 131 patients enrolled, 5 were excluded from the analysis due to protocol violations, so the PP sample was represented by 126 patients $(53,19$, and 54 patients in the LCIG, CSAI, and CU groups) and their related carers.

The demographic characteristics of patients and caregivers, and the main PD clinical characteristics, are shown in Table 1.

Most of carers were female (56\% in LCIG, $79 \%$ in CSAI, and $80 \%$ in CU group), mainly represented by spouse (62\% in LCIG, $68 \%$ in CSAI, and 56\% in CU group) assisting their partner for more than 12 months in most of the cases $(86 \%$ LCIG, 100\% CSAI, and 94\% CU group).

The oral or transdermal medications taken in each group were mainly represented by oral levodopa in 50 out of 53 patients (94\%) and in all the 19 CSAI patients $(100 \%)$ followed by dopamine agonists. The LEDD of each medication was higher in CSAI compared to LCIG group. A similar distribution was seen in $\mathrm{CU}$ group (Table 2). 
Table I Demographic Characteristics of the Patients and Caregivers and PD Clinical Characteristics

\begin{tabular}{|c|c|c|c|c|}
\hline & LCIG (n=53) & CSAI $(n=19)$ & $C U(n=54)$ & $\mathbf{P}$ value $*$, ** \\
\hline $\begin{array}{l}\text { Patients Demographics } \\
\text { Age (years); mean } \pm \text { SD (range) } \\
\text { Male; n (\%) }\end{array}$ & $\begin{array}{l}70.26 \pm 7.1(53-84) \\
25(47 \%)\end{array}$ & $\begin{array}{l}66.0 \pm 6.6(54-77) \\
10(53 \%)\end{array}$ & $\begin{array}{l}69.57 \pm 9.1(42-88) \\
29(54 \%)\end{array}$ & \\
\hline $\begin{array}{l}\text { PD Characteristics } \\
\text { Hoehn \& Yahr in OFF, N (\%) } \\
-1 \\
-1.5 \\
-2 \\
-2.5 \\
-3 \\
-4\end{array}$ & $\begin{array}{l}6(11 \%) \\
37(70 \%) \\
9(17 \%) \\
1(2 \%) \\
0\end{array}$ & $\begin{array}{l}1(5 \%) \\
8(42 \%) \\
8(42 \%) \\
2(11 \%) \\
0\end{array}$ & $\begin{array}{l}0 \\
9(17 \%) \\
36(67 \%) \\
8(15 \%) \\
1(2 \%)\end{array}$ & $\begin{array}{l}* 0.037 \\
* *<0.001\end{array}$ \\
\hline PD duration (years), mean $\pm \mathrm{SD}$, (range) & $16.38 \pm 5.8(7-33)$ & $13.58 \pm 4.1(8-21)$ & $12.83 \pm 5 . \mid(4-35)$ & $* 0.085 * 0.0003$ \\
\hline $\begin{array}{l}\text { Duration of motor fluctuations (years), mean } \pm S D \text {, } \\
\text { (range) }\end{array}$ & $8.36 \pm 4.8(I-26)$ & $5.47 \pm 3.5(2-14)$ & $5.22 \pm 4.1(I-25)$ & $\begin{array}{l}* 0.0099 * * \\
<0.0001\end{array}$ \\
\hline $\begin{array}{l}\text { Caregivers Characteristics } \\
\text { Age (years); mean } \pm \text { SD (range) } \\
\text { Female; } n(\%)\end{array}$ & $\begin{array}{l}59.19 \pm 13.2 \\
(36-84) \\
30(56 \%)\end{array}$ & $\begin{array}{l}60.26 \pm 12.9 \\
(29-78) \\
15(79 \%)\end{array}$ & $\begin{array}{l}55.89 \pm 12.6 \\
(29-85) \\
43(80 \%)\end{array}$ & \\
\hline Daily duration of supporting assistance & $\mathbf{N}=\mathbf{5 3}$ & $N=19$ & $\mathbf{N}=52$ & \\
\hline $\begin{array}{l}\text { Day and night ( } 24 \text { hours) } \\
\text { During daytime } \\
\text { From } 3 \text { to } 6 \text { hours per day }\end{array}$ & $\begin{array}{l}26(49 \%) \\
8(15 \%) \\
19(36 \%)\end{array}$ & $\begin{array}{l}12(63 \%) \\
4(21 \%) \\
3(16 \%)\end{array}$ & $\begin{array}{l}29(56 \%) \\
9(17 \%) \\
14(27 \%)\end{array}$ & $\begin{array}{l}* 0.1783 * 0.2013 \\
* 0.2762 * 0.6155 \\
* 0.3075 * 0.0003\end{array}$ \\
\hline $\begin{array}{l}\text { Caregiver burden } \\
\text { ZBI score (mean } \pm S D)\end{array}$ & $29.6 \pm 14.42$ & $35.8 \pm 20.15$ & $31.4 \pm 16.0$ & $* 0.328 * * 0.535$ \\
\hline
\end{tabular}

Notes: *LCIG vs. CSAI; **LCIG vs. CU; Two-Sample T-test and Wilcoxon Two Sample Test for PD characteristic analysis; Chi Square Test or Fisher Test for daily time spent for the assistance; *LCIG vs. CSAI; **LCIG vs. CU; Bold indicates statistical significance.

Abbreviations: LCIG, levodopa/carbidopa intestinal gel; CSAI, subcutaneous apomorphine infusion; CU, care as usual; SD, standard deviation; N/A, not available; ZBI, Zarit Burden Interview.

The clinical characteristics of patients in each group were extensively described by Tessitore et al. ${ }^{18}$ In LCIG group the absence of daily OFF periods or less than $25 \%$ of the daily time spent in OFF, as assessed by UPDRS-IV item 39, was detected in $81 \%$ of the cases. In the CSAI and $\mathrm{CU}$ groups these percentages were significantly lower (respectively $47 \%$ and $17 \%$; $p=0.037$ LCIG vs. CSAI and $\mathrm{p}<0.001$ LCIG vs. CU).

Table 2 Use of Antiparkinsonian Medications and Corresponding LEDD Before the Implementation of LCIG, CSAI or in Patients Continuing $\mathrm{CU}$

\begin{tabular}{|l|l|l|l|l|l|l|}
\hline & \multicolumn{2}{|l|}{ LCIG (N=50) } & \multicolumn{2}{l|}{ CSAI (N=19) } & \multicolumn{2}{l|}{ CU (N=54) } \\
\cline { 2 - 7 } & N (\%) & $\begin{array}{l}\text { LEDD (mg) (Mean } \pm \\
\text { SD) (Range) }\end{array}$ & N (\%) & $\begin{array}{l}\text { LEDD (mg) (Mean } \pm \\
\text { SD) (Range) }\end{array}$ & N (\%) & $\begin{array}{l}\text { LEDD (mg) (Mean } \pm \\
\text { SD) (Range) }\end{array}$ \\
\hline Oral levodopa & $50(94 \%)$ & $972.5 \pm 417.9(250-2350)$ & $19(100 \%)$ & $1185 \pm 686.3(500-2500)$ & $54(100 \%)$ & $732.6 \pm 230.1(200-1200)$ \\
Dopamine agonists & $33(62 \%)$ & $274.6 \pm 123.9(100-560)$ & $12(63 \%)$ & $437.2 \pm 596.5(105-2000)$ & $36(67 \%)$ & $211.6 \pm 81.0(100-360)$ \\
COMT inhibitors & $23(43 \%)$ & $322.6 \pm 230.8(100-1200)$ & $13(68 \%)$ & $442.7 \pm 403.1(75-1400)$ & $20(37 \%)$ & $548.7 \pm 414.0(132-1400)$ \\
MAO inhibitors & $17(32 \%)$ & $100.0 \pm 0.0(100-100)$ & $3(16 \%)$ & $100 \pm 0(100-100)$ & $19(35 \%)$ & $237.5 \pm 388.9(100-1200)$ \\
Others* & $12(23 \%)$ & $204.3 \pm 108.3(100-360)$ & $1(5 \%)$ & $200(200-200)$ & $15(28 \%)$ & $293.2 \pm 443.9(100-1625)$ \\
\hline
\end{tabular}

Note: *Others= amantadine, apomorphine pen (in LCIG), levodopa/carbidopa/entacapone association 


\section{Caregiver Burden and Questionnaire Assessment}

The mean ZBI score shows a tendency to be lower in LCIG group compared to CSAI or CU group, even if no statistically significant difference was found among groups (Table 1). The boxplot of the ZBI Score showed that LCIG and CU populations have a very similar distribution (LCIG median value 27; IQR 18-42; CU median value 28.5; IQR 19-43; Figure 1). The CSAI ZBI boxplot distribution is slightly wider but not statistically different (CSAI median value 34; IQR 16-52). The aggregated results on ZBI scores "never/rarely" vs. "sometimes/quite frequently/nearly always" did not show a significant difference between groups for each question, except for the question number 6, regarding the negative influence of the assistance on the relationship with other relatives or friends; in this case, a difference was found between LCIG and CSAI ( $\mathrm{p}=0.0269$ ) (Table 3). The following variables were correlated with the ZBI score: "caregiver's change in capability to perform family duties and leisure activities," "caregiver's change in work," "need of professional assistance," "patient's judgment on QoL," and "caregiver's judgment on QoL." "18 The analysis of the distribution of these significant associations is described in Figure 2A and B. The UPDRS-IV item 39 and the H\&Y stage did not show any association with the ZBI score (Figure 2C).

Data from the questionnaires to caregivers showed that carers assisting patients in treatment with LCIG have more time to perform family or household duties ( $\mathrm{p}=0.0022$ ), or to engage leisure activities $(\mathrm{p}=0.0073)$ compared to those assisting patients in treatment with $\mathrm{CU}$, while no difference was found compared to CSAI group (Table 3). Furthermore, caregivers of patients treated with LCIG perceived that their assisted relatives were more independent in taking a bath or shower without assistance compared to what perceived by caregivers of CSAI and CU patients ( $\mathrm{p}=0.00198$ vs. CSAI and $\mathrm{p}=0.0586$ vs. CU) and more able to move and walk without assistance $(\mathrm{p}=0.0002)$ compared to $\mathrm{CU}$ population (Table 4).

A tendency to an improvement in patient's autonomy for staying alone at home was observed in the CSAI group (35\%) compared to LCIG (19\%) and CU groups (5\%) even if the difference was not significant. Moreover, caregivers assisting CU patients found more difficulties in helping other relatives ( $21 \%$ of the cases) compared to care partners of LCIG $(8 \%)$ or CSAI patients $(0 \%)$ (Table 4).

\section{Quality of Life Perception and Relation to Burden}

In Figure 2B we show the association between the judgment on QoL expressed by both the patients and their caregivers with the corresponding ZBI score in each treatment group. A lower ZBI score was detected in patients and carers having a better QoL perception.

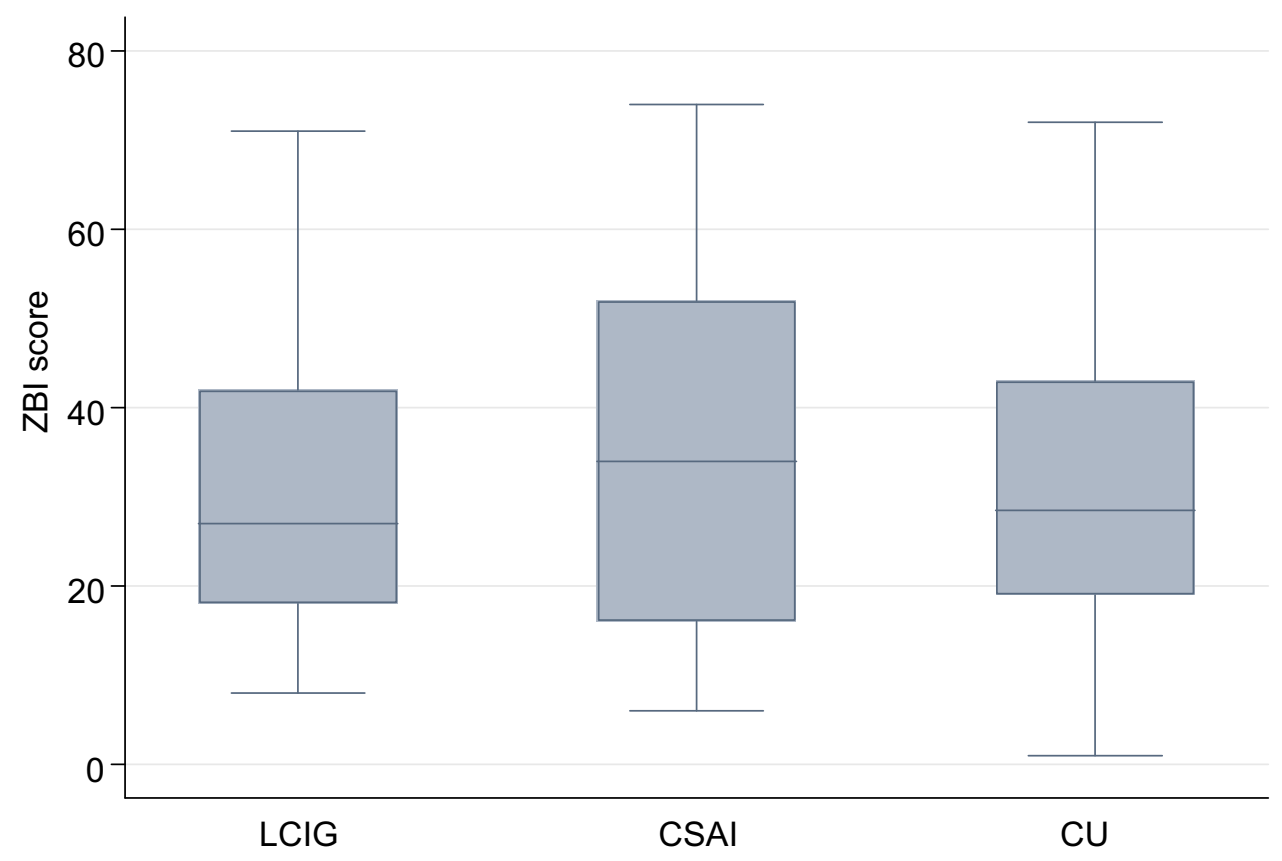

Figure I Frequency of symptoms reported by caregivers (A) and kind of mood change (B) in each group. 
Table 3 Aggregated Results for ZBI Scores in the Three Groups of Treatment. The Total Percentage in Each Group Were Computed Excluding the Missing Data (Not Reported in This Table)

\begin{tabular}{|c|c|c|c|c|c|c|c|}
\hline \multirow{3}{*}{$\begin{array}{l}\text { Question } \\
\text { I. Do you feel that your relative asks for more } \\
\text { help than he/she needs? }\end{array}$} & \multirow{3}{*}{$\begin{array}{l}\text { Categorized } \\
\text { Answers } \\
\text { Never+Rarely } \\
\text { Sometime } \\
\text { +Quite frequently } \\
\text { +nearly always }\end{array}$} & \multirow{3}{*}{$\begin{array}{l}\text { LCIG; } \\
\text { N (\%) } \\
23(43.40) \\
30(56.60)\end{array}$} & \multirow{3}{*}{$\begin{array}{l}\text { CSAl; } \\
\text { N (\%) } \\
4(21.05) \\
15(78.95)\end{array}$} & \multirow{3}{*}{$\begin{array}{l}\text { CU; } \\
\text { N (\%) } \\
27(50) \\
27(50)\end{array}$} & \multirow{2}{*}{\multicolumn{3}{|c|}{$\begin{array}{l}\text { Comparison Between Treatment Groups } \\
\text { P value }\end{array}$}} \\
\hline & & & & & & & \\
\hline & & & & & $\begin{array}{l}\mathrm{LCIG} \text { versus } \mathrm{CSAI} \\
\mathrm{LCIG} \text { versus } \mathrm{CU}\end{array}$ & $\begin{array}{l}\text { Fisher Test } \\
\text { Chi-Square Test }\end{array}$ & $\begin{array}{l}0.1033 \\
0.4936\end{array}$ \\
\hline $\begin{array}{l}\text { 2. Do you feel that because of the time you spend } \\
\text { with your relative that you do not have enough } \\
\text { time for yourself? }\end{array}$ & $\begin{array}{l}\text { Never+Rarely } \\
\text { Sometime+Quite } \\
\text { frequently+nearly } \\
\text { always }\end{array}$ & $\begin{array}{l}21(40.38) \\
31(59.62)\end{array}$ & $\begin{array}{l}7(36.84) \\
12(63.16)\end{array}$ & $\begin{array}{l}23(42.59) \\
3 I(57.4 I)\end{array}$ & $\begin{array}{l}\text { LCIG versus CSAI } \\
\text { LCIG versus } C U\end{array}$ & $\begin{array}{l}\text { Chi-Square Test } \\
\text { Chi-Square Test }\end{array}$ & $\begin{array}{l}0.7869 \\
0.8176\end{array}$ \\
\hline $\begin{array}{l}\text { 3. Do you feel stressed between caring for your } \\
\text { relative and trying to meet other responsibilities } \\
\text { for your family or work? }\end{array}$ & $\begin{array}{l}\text { Never+Rarely } \\
\text { Sometime+Quite } \\
\text { frequently+nearly } \\
\text { always }\end{array}$ & $\begin{array}{l}24(45.28) \\
29(54.72)\end{array}$ & $\begin{array}{l}5(26.32) \\
14(73.68)\end{array}$ & $\begin{array}{l}16(29.63) \\
38(70.37)\end{array}$ & $\begin{array}{l}\text { LCIG versus } C S A I \\
L C I G \text { versus } C U\end{array}$ & $\begin{array}{l}\text { Chi-Square Test } \\
\text { Chi-Square Test }\end{array}$ & $\begin{array}{l}0.1481 \\
0.0943\end{array}$ \\
\hline $\begin{array}{l}\text { 4. Do you feel embarrassed over your relative's } \\
\text { behavior? }\end{array}$ & $\begin{array}{l}\text { Never+Rarely } \\
\text { Sometime+Quite } \\
\text { frequently+nearly } \\
\text { always }\end{array}$ & $\begin{array}{l}44(83.02) \\
9(16.98)\end{array}$ & $\begin{array}{l}14(73.68) \\
5(26.32)\end{array}$ & $\begin{array}{l}43(79.63) \\
11(20.37)\end{array}$ & $\begin{array}{l}\text { LCIG versus } C S A I \\
L C I G \text { versus } C U\end{array}$ & $\begin{array}{l}\text { Chi-Square Test } \\
\text { Chi-Square Test }\end{array}$ & $\begin{array}{l}0.3777 \\
0.6530\end{array}$ \\
\hline $\begin{array}{l}\text { 5. Do you feel angry when you are around your } \\
\text { relative? }\end{array}$ & $\begin{array}{l}\text { Never+Rarely } \\
\text { Sometime+Quite } \\
\text { frequently+nearly } \\
\text { always }\end{array}$ & $\begin{array}{l}39(73.58) \\
14(26.42)\end{array}$ & $\begin{array}{l}15(78.95) \\
4(21.05)\end{array}$ & $\begin{array}{l}36(67.92) \\
17(32.08)\end{array}$ & $\begin{array}{l}\mathrm{LCIG} \text { versus } \mathrm{CSAI} \\
\mathrm{LCIG} \text { versus } \mathrm{CU}\end{array}$ & $\begin{array}{l}\text { Fisher Test } \\
\text { Chi-Square Test }\end{array}$ & $\begin{array}{l}0.7639 \\
0.5218\end{array}$ \\
\hline $\begin{array}{l}\text { 6. Do you feel that your relative currently affects } \\
\text { your relationship with other family members or } \\
\text { friends in a negative way? }\end{array}$ & $\begin{array}{l}\text { Never+Rarely } \\
\text { Sometime+Quite } \\
\text { frequently+nearly } \\
\text { always }\end{array}$ & $\begin{array}{l}44(83.02) \\
9(16.98)\end{array}$ & $\begin{array}{l}\text { II (57.89) } \\
8(42.11)\end{array}$ & $\begin{array}{l}39(72.22) \\
15(27.78)\end{array}$ & $\begin{array}{l}\mathrm{LCIG} \text { versus } \mathrm{CSAI} \\
\mathrm{LCIG} \text { versus } \mathrm{CU}\end{array}$ & $\begin{array}{l}\text { Chi-Square Test } \\
\text { Chi-Square Test }\end{array}$ & $\begin{array}{l}\mathbf{0 . 0 2 6 9} \\
0.1807\end{array}$ \\
\hline $\begin{array}{l}\text { 7. Are you afraid what the future holds for your } \\
\text { relative? }\end{array}$ & $\begin{array}{l}\text { Never+Rarely } \\
\text { Sometime+Quite } \\
\text { frequently+nearly } \\
\text { always }\end{array}$ & $\begin{array}{l}14(26.42) \\
39(73.58)\end{array}$ & $\begin{array}{l}3(15.79) \\
16(84.21)\end{array}$ & $\begin{array}{l}10(18.52) \\
44(81.48)\end{array}$ & $\begin{array}{l}\text { LCIG versus CSAI } \\
\text { LCIG versus } C U\end{array}$ & $\begin{array}{l}\text { Fisher Test } \\
\text { Chi-Square Test }\end{array}$ & $\begin{array}{l}0.5307 \\
0.3275\end{array}$ \\
\hline $\begin{array}{l}\text { 8. Do you feel your relative is dependent upon } \\
\text { you? }\end{array}$ & $\begin{array}{l}\text { Never+Rarely } \\
\text { Sometime+Quite } \\
\text { frequently+nearly } \\
\text { always }\end{array}$ & $\begin{array}{l}8(15.38) \\
44(84.62)\end{array}$ & $\begin{array}{l}4(22.22) \\
14(77.78)\end{array}$ & $\begin{array}{l}9(16.67) \\
45(83.33)\end{array}$ & $\begin{array}{l}\text { LCIG versus CSAI } \\
\text { LCIG versus } C U\end{array}$ & $\begin{array}{l}\text { Fisher Test } \\
\text { Chi-Square Test }\end{array}$ & $\begin{array}{l}0.4903 \\
0.8573\end{array}$ \\
\hline $\begin{array}{l}\text { 9. Do you feel strained when you are around your } \\
\text { relative? }\end{array}$ & $\begin{array}{l}\text { Never+Rarely } \\
\text { Sometime+Quite } \\
\text { frequently+nearly } \\
\text { always }\end{array}$ & $\begin{array}{l}38(71.70) \\
15(28.30)\end{array}$ & $\begin{array}{l}10(52.63) \\
9(47.37)\end{array}$ & $\begin{array}{l}32(59.26) \\
22(40.74)\end{array}$ & $\begin{array}{l}\text { LCIG versus } C S A I \\
L C I G \text { versus } C U\end{array}$ & $\begin{array}{l}\text { Chi-Square Test } \\
\text { Chi-Square Test }\end{array}$ & $\begin{array}{l}0.1304 \\
0.1762\end{array}$ \\
\hline $\begin{array}{l}\text { 10. Do you feel your health has suffered because } \\
\text { of your involvement with your relative? }\end{array}$ & $\begin{array}{l}\text { Never+Rarely } \\
\text { Sometime+Quite } \\
\text { frequently+nearly } \\
\text { always }\end{array}$ & $\begin{array}{l}31(58.49) \\
22(41.51)\end{array}$ & $\begin{array}{l}12(63.16) \\
7(36.84)\end{array}$ & $\begin{array}{l}24(44.44) \\
30(55.56)\end{array}$ & $\begin{array}{l}\text { LCIG versus CSAI } \\
\text { LCIG versus } C U\end{array}$ & $\begin{array}{l}\text { Chi-Square Test } \\
\text { Chi-Square Test }\end{array}$ & $\begin{array}{l}0.7219 \\
0.1461\end{array}$ \\
\hline $\begin{array}{l}\text { II. Do you feel that you do not have as much } \\
\text { privacy as you would like because of your relative? }\end{array}$ & $\begin{array}{l}\text { Never+Rarely } \\
\text { Sometime+Quite } \\
\text { frequently+nearly } \\
\text { always }\end{array}$ & $\begin{array}{l}25(47.17) \\
28(52.83)\end{array}$ & $\begin{array}{l}4(22.22) \\
14(77.78)\end{array}$ & $\begin{array}{l}24(44.44) \\
30(55.56)\end{array}$ & $\begin{array}{l}\text { LCIG versus CSAI } \\
\text { LCIG versus } C U\end{array}$ & $\begin{array}{l}\text { Fisher Test } \\
\text { Chi-Square Test }\end{array}$ & $\begin{array}{l}0.0954 \\
0.7773\end{array}$ \\
\hline
\end{tabular}

(Continued) 
Table 3 (Continued).

\begin{tabular}{|c|c|c|c|c|c|c|c|}
\hline \multirow{3}{*}{$\begin{array}{l}\text { Question } \\
\text { 12. Do you feel that your social life has suffered } \\
\text { because you are caring for your relative? }\end{array}$} & \multirow{3}{*}{$\begin{array}{l}\begin{array}{l}\text { Categorized } \\
\text { Answers }\end{array} \\
\begin{array}{l}\text { Never+Rarely } \\
\text { Sometime+Quite } \\
\text { frequently+nearly } \\
\text { always }\end{array}\end{array}$} & \multirow{3}{*}{$\begin{array}{l}\text { LCIG; } \\
\text { N (\%) } \\
34(64.15) \\
19(35.85)\end{array}$} & \multirow{3}{*}{$\begin{array}{l}\text { CSAl; } \\
\text { N (\%) } \\
10(52.63) \\
9(47.37)\end{array}$} & \multirow{3}{*}{$\begin{array}{l}\text { CU; } \\
\text { N (\%) } \\
36(66.67) \\
18(33.33)\end{array}$} & \multirow{2}{*}{\multicolumn{3}{|c|}{$\begin{array}{l}\text { Comparison Between Treatment Groups } \\
\text { P value }\end{array}$}} \\
\hline & & & & & & & \\
\hline & & & & & $\begin{array}{l}\text { LCIG versus CSAI } \\
L C I G \text { versus } C U\end{array}$ & $\begin{array}{l}\text { Chi-Square Test } \\
\text { Chi-Square Test }\end{array}$ & $\begin{array}{l}0.3769 \\
0.7844\end{array}$ \\
\hline $\begin{array}{l}\text { 13. Do you feel uncomfortable about having } \\
\text { friends over because of your relative? }\end{array}$ & $\begin{array}{l}\text { Never+Rarely } \\
\text { Sometime+Quite } \\
\text { frequently+nearly } \\
\text { always }\end{array}$ & $\begin{array}{l}48(90.57) \\
5(9.43)\end{array}$ & $\begin{array}{l}15(78.95) \\
4(21.05)\end{array}$ & $\begin{array}{l}45(83.33) \\
9(16.67)\end{array}$ & $\begin{array}{l}\mathrm{LCIG} \text { versus } \mathrm{CSAI} \\
\mathrm{LCIG} \text { versus } \mathrm{CU}\end{array}$ & $\begin{array}{l}\text { Fisher Test } \\
\text { Chi-Square Test }\end{array}$ & $\begin{array}{l}0.2311 \\
0.2673\end{array}$ \\
\hline $\begin{array}{l}\text { 14. Do you feel that your relative seems to expect } \\
\text { you to take care of him/her as if you were the } \\
\text { only one he/she could depend on? }\end{array}$ & $\begin{array}{l}\text { Never+Rarely } \\
\text { Sometime+Quite } \\
\text { frequently+nearly } \\
\text { always }\end{array}$ & $\begin{array}{l}15(28.30) \\
38(71.70)\end{array}$ & $\begin{array}{l}8(44.44) \\
10(55.56)\end{array}$ & $\begin{array}{l}20(37.04) \\
34(62.96)\end{array}$ & $\begin{array}{l}\text { LCIG versus CSAI } \\
\text { LCIG versus } C U\end{array}$ & $\begin{array}{l}\text { Chi-Square Test } \\
\text { Chi-Square Test }\end{array}$ & $\begin{array}{l}0.2061 \\
0.3356\end{array}$ \\
\hline $\begin{array}{l}\text { 15. Do you feel that you do not have enough } \\
\text { money to care for your relative in addition to the } \\
\text { rest of your expenses? }\end{array}$ & $\begin{array}{l}\text { Never+Rarely } \\
\text { Sometime+Quite } \\
\text { frequently+nearly } \\
\text { always }\end{array}$ & $\begin{array}{l}33(62.26) \\
20(37.74)\end{array}$ & $\begin{array}{l}9(47.37) \\
10(52.63)\end{array}$ & $\begin{array}{l}24(45.28) \\
29(54.72)\end{array}$ & $\begin{array}{l}\text { LCIG versus CSAI } \\
L C I G \text { versus } C U\end{array}$ & $\begin{array}{l}\text { Chi-Square Test } \\
\text { Chi-Square Test }\end{array}$ & $\begin{array}{l}0.2585 \\
0.0795\end{array}$ \\
\hline $\begin{array}{l}\text { 16. Do you feel that you will be unable to take } \\
\text { care of your relative much longer? }\end{array}$ & $\begin{array}{l}\text { Never+Rarely } \\
\text { Sometime+Quite } \\
\text { frequently+nearly } \\
\text { always }\end{array}$ & $\begin{array}{l}31(58.49) \\
22(41.51)\end{array}$ & $\begin{array}{l}12(66.67) \\
6(33.33)\end{array}$ & $\begin{array}{l}36(66.67) \\
18(33.33)\end{array}$ & $\begin{array}{l}\text { LCIG versus } C S A I \\
L C I G \text { versus } C U\end{array}$ & $\begin{array}{l}\text { Chi-Square Test } \\
\text { Chi-Square Test }\end{array}$ & $\begin{array}{l}0.5397 \\
0.3821\end{array}$ \\
\hline $\begin{array}{l}\text { 17. Do you feel you have lost control of your life } \\
\text { since your relative's illness? }\end{array}$ & $\begin{array}{l}\text { Never+Rarely } \\
\text { Sometime+Quite } \\
\text { frequently+nearly } \\
\text { always }\end{array}$ & $\begin{array}{l}38(71.70) \\
15(28.30)\end{array}$ & $\begin{array}{l}10(52.63) \\
9(47.37)\end{array}$ & $\begin{array}{l}31(57.41) \\
23(42.59)\end{array}$ & $\begin{array}{l}\mathrm{LCIG} \text { versus } \mathrm{CSAI} \\
\mathrm{LCIG} \text { versus } \mathrm{CU}\end{array}$ & $\begin{array}{l}\text { Chi-Square Test } \\
\text { Chi-Square Test }\end{array}$ & $\begin{array}{l}0.1304 \\
0.1225\end{array}$ \\
\hline $\begin{array}{l}\text { 18. Do you wish you could leave the care of your } \\
\text { relative to someone else? }\end{array}$ & $\begin{array}{l}\text { Never+Rarely } \\
\text { Sometime+Quite } \\
\text { frequently+nearly } \\
\text { always }\end{array}$ & $\begin{array}{l}36(67.92) \\
17(32.08)\end{array}$ & $\begin{array}{l}9(50.00) \\
9(50.00)\end{array}$ & $\begin{array}{l}36(66.67) \\
18(33.33)\end{array}$ & $\begin{array}{l}\text { LCIG versus CSAI } \\
L C I G \text { versus } C U\end{array}$ & $\begin{array}{l}\text { Chi-Square Test } \\
\text { Chi-Square Test }\end{array}$ & $\begin{array}{l}0.1726 \\
0.8897\end{array}$ \\
\hline $\begin{array}{l}\text { 19. Do you feel uncertain about what to do about } \\
\text { your relative? }\end{array}$ & $\begin{array}{l}\text { Never+Rarely } \\
\text { Sometime+Quite } \\
\text { frequently+nearly } \\
\text { always }\end{array}$ & $\begin{array}{l}35(66.04) \\
18(33.96)\end{array}$ & $\begin{array}{l}8(50.00) \\
8(50.00)\end{array}$ & $\begin{array}{l}33(61.11) \\
21(38.89)\end{array}$ & $\begin{array}{l}\mathrm{LCIG} \text { versus } \mathrm{CSAI} \\
\mathrm{LCIG} \text { versus } \mathrm{CU}\end{array}$ & $\begin{array}{l}\text { Chi-Square Test } \\
\text { Chi-Square Test }\end{array}$ & $\begin{array}{l}0.2460 \\
0.5965\end{array}$ \\
\hline $\begin{array}{l}\text { 20. Do you feel you should be doing more for } \\
\text { your relative? }\end{array}$ & $\begin{array}{l}\text { Never+Rarely } \\
\text { Sometime+Quite } \\
\text { frequently+nearly } \\
\text { always }\end{array}$ & $\begin{array}{l}23(43.40) \\
30(56.60)\end{array}$ & $\begin{array}{l}9(43.37) \\
10(52.63)\end{array}$ & $\begin{array}{l}29(54.72) \\
31(57.41)\end{array}$ & $\begin{array}{l}\mathrm{LCIG} \text { versus } \mathrm{CSAI} \\
\mathrm{LCIG} \text { versus } \mathrm{CU}\end{array}$ & $\begin{array}{l}\text { Chi-Square Test } \\
\text { Chi-Square Test }\end{array}$ & $\begin{array}{l}0.7650 \\
0.2437\end{array}$ \\
\hline $\begin{array}{l}21 \text {. Do you feel you could do a better job in caring } \\
\text { for your relative? }\end{array}$ & $\begin{array}{l}\text { Never+Rarely } \\
\text { Sometime+Quite } \\
\text { frequently+nearly } \\
\text { always }\end{array}$ & $\begin{array}{l}27(50.94) \\
26(49.06)\end{array}$ & $\begin{array}{l}10(52.63) \\
9(47.37)\end{array}$ & $\begin{array}{l}3 I(57.4 I) \\
23(42.59)\end{array}$ & $\begin{array}{l}\text { LCIG versus CSAI } \\
\text { LCIG versus CU }\end{array}$ & $\begin{array}{l}\text { Chi-Square Test } \\
\text { Chi-Square Test }\end{array}$ & $\begin{array}{l}0.8995 \\
0.5022\end{array}$ \\
\hline $\begin{array}{l}\text { 22. Overall, how burdened do you feel in caring } \\
\text { for your relative? }\end{array}$ & $\begin{array}{l}\text { Not at all+ A little } \\
\text { Rather+A lot } \\
\text { +extremely }\end{array}$ & $\begin{array}{l}32(62.75) \\
19(37.25)\end{array}$ & $\begin{array}{l}9(47.37) \\
10(52.63)\end{array}$ & $\begin{array}{l}30(55.56) \\
24(44.44)\end{array}$ & $\begin{array}{l}\text { LCIG versus CSAI } \\
L C I G \text { versus } C U\end{array}$ & $\begin{array}{l}\text { Chi-Square Test } \\
\text { Chi-Square Test }\end{array}$ & $\begin{array}{l}0.2455 \\
0.4540\end{array}$ \\
\hline
\end{tabular}

Notes: Reproduced with permission from Zarit SH and Zarit JM; $1983 .{ }^{34}$ (www.mapi-trust.org, https://eprovide.mapi-trust.org/). This is not the official format of the ZBI, only a table to stratify the results. Bold value indicates statistical significance. 
The mean value of the self-assessment and the single values in each treatment group expressed both by patients and their carers on their current QoL compared with the previous CU is represented in Figure 3.

In our previously published data, we showed that patient's QoL was significantly better in the LCIG and CSAI groups compared with the CU group according to PDQ-8 scores. ${ }^{18}$ In this further analysis, it has been observed a moderate association between the mean daily OFF duration and the mean PDQ-8 score (Spearman correlation coefficient $=0.4)$, higher for the $\mathrm{CU}$ group $(0.41)$ and lower for CSAI and LCIG (respectively, 0.34 and 0.21 ).

The assessment of the caregivers' condition in the previous 6 months revealed that approximately $50 \%$ in each group had a mood variation. Mood improved in $38 \%$ and $30 \%$ of the LCIG and CSAI carers, while this improvement was observed only in $9 \%$ of the CU caregivers without statistically significant differences between groups (Figure 4A and B). One third of caregivers in each group and more than $40 \%$ in LCIG and CSAI groups showed respectively sleep disturbances and anxiety (Figure 4A).

\section{Discussion}

This analysis on the PREDICT study data is focused on the caregivers QoL and the qualitative assessment of their burden due to the daily assistance of advanced PD patients.

The study has shown that caregivers assisting patients with LCIG showed a lower burden compared to the other groups with a similar variability range as for example carers assisting $\mathrm{CU}$ patients. Data from the analysis of the single items of the ZBI questionnaire reported that caregivers are mainly affected by the thought that the patient can have a negative influence on their relationships with other family members or friends.

The mean ZBI score shown in our previous paper was in line with the ZBI range reported in PD caregivers in previous studies. ${ }^{11,25}$ Some previously published data reported a lower mean ZBI score of $21.87 \pm 16.59,{ }^{26} 14.4 \pm 12.7,{ }^{10}$ and $15.2 \pm$ 9.0, ${ }^{27}$ but the majority of PD patients were in H\&Y stage 1 or 2. In this new data presentation, although not statistically significant, we showed a trend towards a wider ZBI score range for $\mathrm{CU}$ compared to LCIG and CSAI suggesting a possible more stable distress condition of these latter caregivers. It has been recently reported that disease duration, disease stage, activities of daily living and motor symptoms were the most commonly explored variables but not in all studies these were found to be predictors of caregiver burden. ${ }^{2,8,28}$ Recently, it has been shown that the intensity of caregiving (informal hours and years of caregiving) were correlated with caregiver burden, ${ }^{29}$ while in another study caregiving duration was weakly associated with burden and depression. ${ }^{30}$ In our study, patient's QoL, caregiver's change in ability to perform family duties and leisure activities, caregiver's change in work, the need of professional assistance, and patient and caregiver judgment on QoL represented the main influencing factors on carers burden. Differently, H\&Y staging, PD duration, duration of motor fluctuations, the presence of PD associated symptoms, or the duration of caregiver assistance showed no significant association with the caregiver's burden.

Even though a longer disease duration of the LCIG patients (16.3 years) compared with CSAI and CU caregivers (13.6 and 12.8 years, respectively) and a longer duration of motor fluctuations ( 8.3 vs. 5.4 and 5.2 years) were reported in the previously published paper on PREDICT, the level of burden in LCIG caregivers was lower and of milder severity compared to CSAI and CU caregivers. ${ }^{18}$ Moreover, in our research, a high percentage of caregivers in each group assisted their relative both during the day and the night (from $49 \%$ to $63 \%$ ) and almost all of them for more than 12 months. This percentage is consistent with the percentage of day and night assistance of $40 \%$ recently reported by Grun et al. ${ }^{27}$ In fact, it is interesting to note that in the paper published by Martinez-Martin et al, even if a lower percentage of caregivers gave their assistance during day and night (22.5\%), a similar level of burden was found (mean ZBI score 26.5 $\pm 18.7) .{ }^{3}$ The 24 hours spent by most of caregivers in our study, could have influenced their quality of sleep. In fact, one third of the caregivers in each group (from 30\% to $36 \%$ ) declared that they complained of sleep disturbances and this data is consistent with that published by Grun et al where $41 \%$ of caregivers showed a night sleep disrupted for nocturnal care with a ZBI score of $25.8 \pm 17.1 .^{27}$

In our study we showed that approximately $50 \%$ of the caregivers declared a mood change in the last 6 months and the mood was worsened in the majority of the CU caregivers, while it seems that LCIG and CSAI could have less impact on the mood changes. This is an important issue considering that it has been recently reported that the mood of the caregiver is directly linked 


\section{A}

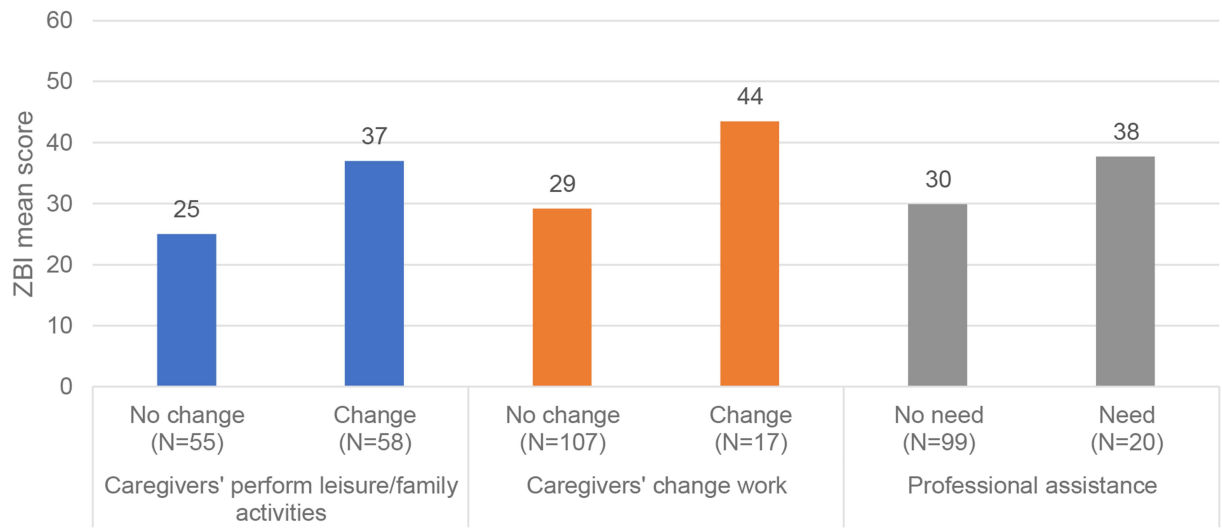

B

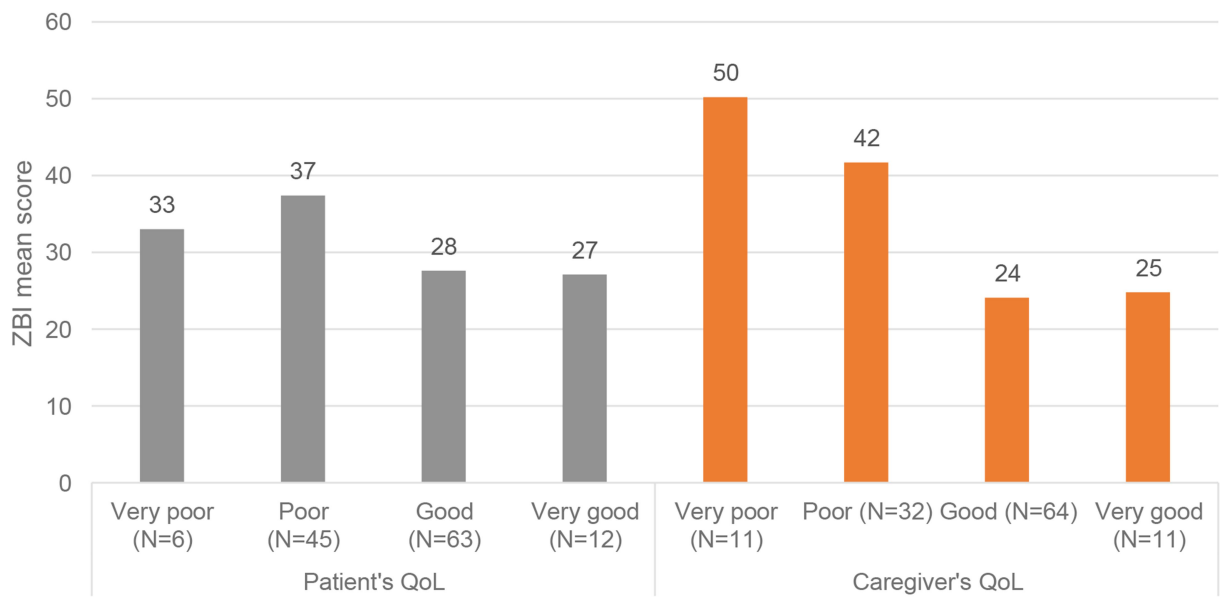

C

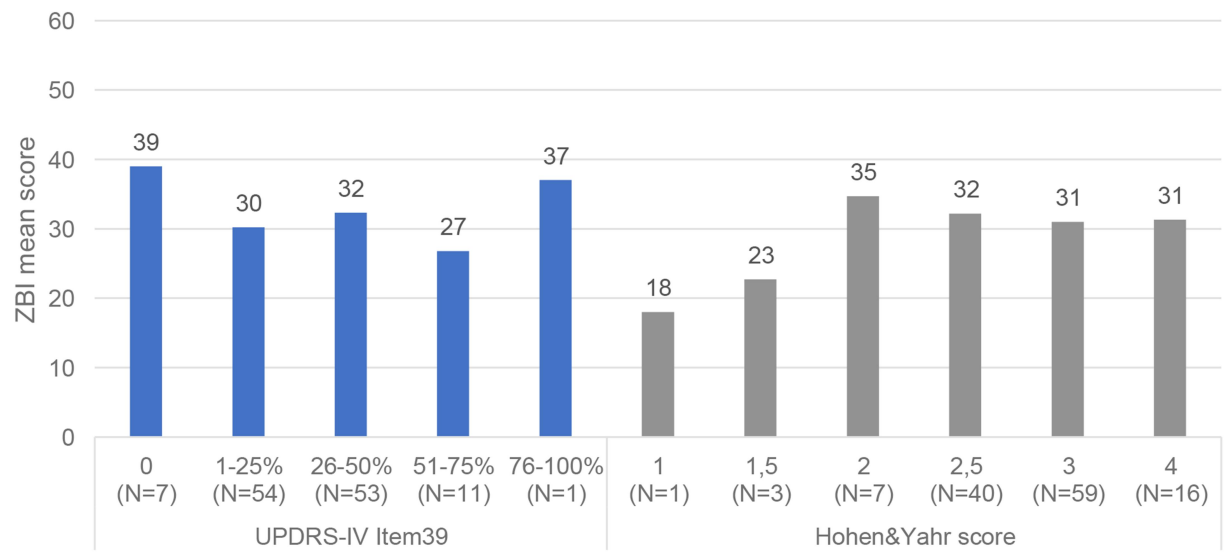

Figure 2 ZBI scores distributions according to caregivers' daily activities and working change, need for professional assistance (A), patient and caregiver quality of life judgment (B), and according to UPDRS-IV item 39 and H\&Y stage (C).

Notes: Level of significance assessed by ANOVA; $(\mathbf{A}) \mathrm{p}<0.001$ for caregiver's leisure activities, $\mathrm{p}=0.00 \mathrm{I}$ for caregiver's changing work and $\mathrm{p}=0.019$ for need of professional assistance; (B) $\mathrm{p}=0.010$ for patient's QoL and $\mathrm{p}<0.00$ I for caregiver's QoL; (C) $\mathrm{p}=\mathrm{NS}$ for UPDRS-IV item 39 and H\&Y score.

to his/her burden. ${ }^{27}$ We have also shown that the frequency of caregiver depression was similarly distributed among groups and with a lower frequency compared to some previously published papers where a percentage between $40 \%$ and $50 \%$ of caregivers reported depression. $^{5,27,31}$ The level of caregiver anxiety in our 
Table 4 Questionnaire Administered to Caregiver in Each Group

\begin{tabular}{|c|c|c|c|c|c|}
\hline \multicolumn{2}{|l|}{ Since the Patient Has Been Optimized with the Actual Therapy: } & \multirow{2}{*}{$\begin{array}{l}\text { LCIG } \\
(N=53) \\
N(\%)\end{array}$} & \multirow{2}{*}{$\begin{array}{l}\text { CSAI } \\
(N=19) \\
N(\%)\end{array}$} & \multirow{2}{*}{$\begin{array}{l}\mathrm{CU} \\
(\mathrm{N}=54) \\
\mathrm{N}(\%)\end{array}$} & \multirow{2}{*}{$\begin{array}{l}\text { Comparisor } \\
\text { Between } \\
\text { Treatment } \\
\text { Groups } \\
\text { P value }\end{array}$} \\
\hline & & & & & \\
\hline $\begin{array}{l}\text { The assistance you provide has given you sufficient time to perform } \\
\text { your family or household duties (i.e., shopping, laundry, house cleaning, } \\
\text { cooking)? }\end{array}$ & $\begin{array}{l}\text { Yes, more than before } \\
\text { Yes, the same as before } \\
\text { Yes, with more difficulties } \\
\text { than before } \\
\text { No }\end{array}$ & $\begin{array}{l}17(32.08) \\
22(41.51) \\
12(22.64) \\
2(3.77)\end{array}$ & $\begin{array}{l}5(26.32) \\
6(31.58) \\
6(31.58) \\
2(10.53)\end{array}$ & $\begin{array}{l}2(4.76) \\
18(42.86) \\
19(45.24) \\
3(7.14)\end{array}$ & $\begin{array}{l}0.5077^{*} \\
\mathbf{0 . 0 0 2 2} * *\end{array}$ \\
\hline $\begin{array}{l}\text { The assistance you provide allows you to help parents and other } \\
\text { relatives? }\end{array}$ & $\begin{array}{l}\text { Yes, more than before } \\
\text { Yes, the same as before } \\
\text { Yes, with more difficulties } \\
\text { than before } \\
\text { No }\end{array}$ & $\begin{array}{l}5(9.62) \\
26(50.00) \\
4(7.69) \\
17(32.69)\end{array}$ & $\begin{array}{l}3(16.67) \\
8(44.44) \\
0(0) \\
7(38.89)\end{array}$ & $\begin{array}{l}\text { I (2.33) } \\
15(34.88) \\
9(20.93) \\
18(41.86)\end{array}$ & $\begin{array}{l}0.6160^{*} \\
0.0818^{* *}\end{array}$ \\
\hline $\begin{array}{l}\text { Do you manage, in view of the assistance you provide, to engage in } \\
\text { leisure activities? }\end{array}$ & $\begin{array}{l}\text { Yes, more than before } \\
\text { Yes, the same as before } \\
\text { Yes, with more difficulties } \\
\text { than before } \\
\text { No }\end{array}$ & $\begin{array}{l}10(19.23) \\
18(34.62) \\
10(19.23) \\
14(26.92)\end{array}$ & $\begin{array}{l}7(36.84) \\
3(15.79) \\
2(10.53) \\
7(36.84)\end{array}$ & $\begin{array}{l}1(2.38) \\
8(19.05) \\
13(30.95) \\
20(47.62)\end{array}$ & $\begin{array}{l}0.2197^{*} \\
0.0073 * *\end{array}$ \\
\hline Can your relative stay home alone while family members are out? & $\begin{array}{l}\text { Yes, more than before } \\
\text { Yes, the same as before } \\
\text { Yes, with more difficulties } \\
\text { than before } \\
\text { No }\end{array}$ & $\begin{array}{l}10(18.87) \\
15(28.30) \\
7(13.21) \\
21(39.62)\end{array}$ & $\begin{array}{l}6(35.29) \\
6(35.29) \\
3(17.65) \\
2(11.76)\end{array}$ & $\begin{array}{l}2(4.65) \\
18(41.86) \\
8(18.60) \\
15(34.88)\end{array}$ & $\begin{array}{l}0.1394^{*} \\
0.1220^{* *}\end{array}$ \\
\hline $\begin{array}{l}\text { Does your relative need assistance in using public transport or driving } \\
\text { a car? }\end{array}$ & $\begin{array}{l}\text { Yes, more than before } \\
\text { Yes, the same as before } \\
\text { Yes, with more difficulties } \\
\text { than before } \\
\text { No }\end{array}$ & $\begin{array}{l}16(30.77) \\
21(40.38) \\
7(13.46) \\
8(15.38)\end{array}$ & $\begin{array}{l}6(33.33) \\
9(50.00) \\
1(5.56) \\
2(11.11)\end{array}$ & $\begin{array}{l}15(34.88) \\
9(20.93) \\
10(23.26) \\
9(20.93)\end{array}$ & $\begin{array}{l}0.8578^{*} \\
0.2028^{* *}\end{array}$ \\
\hline Does your relative take a bath/shower without assistance? & $\begin{array}{l}\text { Yes, more than before } \\
\text { Yes, the same as before } \\
\text { Yes, with more difficulties } \\
\text { than before } \\
\text { No }\end{array}$ & $\begin{array}{l}9(16.98) \\
13(24.53) \\
4(7.55) \\
27(50.94)\end{array}$ & $\begin{array}{l}8(42.11) \\
5(26.32) \\
3(15.79) \\
3(15.79)\end{array}$ & $\begin{array}{l}2(4.76) \\
17(40.48) \\
7(16.67) \\
16(38.10)\end{array}$ & $\begin{array}{l}0.0198 * \\
0.0586 * *\end{array}$ \\
\hline Is your relative able to move and walk without assistance? & $\begin{array}{l}\text { Yes, more than before } \\
\text { Yes, the same as before } \\
\text { Yes, with more difficulties } \\
\text { than before } \\
\text { No }\end{array}$ & $\begin{array}{l}20(37.74) \\
13(24.53) \\
8(15.09) \\
12(22.64)\end{array}$ & $\begin{array}{l}8(42.11) \\
2(10.53) \\
4(21.05) \\
5(26.32)\end{array}$ & $\begin{array}{l}1(2.38) \\
16(38.10) \\
13(30.95) \\
12(28.57)\end{array}$ & $\begin{array}{l}0.6532 * \\
0.0002 * *\end{array}$ \\
\hline
\end{tabular}

Notes: * ${ }^{\mathrm{LCIG}}$ vs. CSAI (Fisher test); ** LCIG vs. CU (Fisher test); Bold indicates statistical significance.

research was consistent with Grun et al, who reported that anxiety was present in $42 \%$ of the caregivers ${ }^{27}$ and slightly higher than the $26 \%$ of a more recent observation. ${ }^{10}$ Even if in our study these observations do not derive from validated questionnaires they are novel real-life descriptive data of carer mental and physical well-being, and they seem to support the influence of physical and mental status on caregiver's QoL.
This evidence is particularly important, because both depression and anxiety in the caregivers are strong predictors of caregiver's burden ${ }^{3,31}$ and depression has a significant negative effect both on caregiver burden and on health-related QoL. ${ }^{2}$ It has recently been reported that after 6 months of LCIG treatment there was a significant improvement of anxiety, depression, level of care burden and QoL, especially in the domains related to social life, 

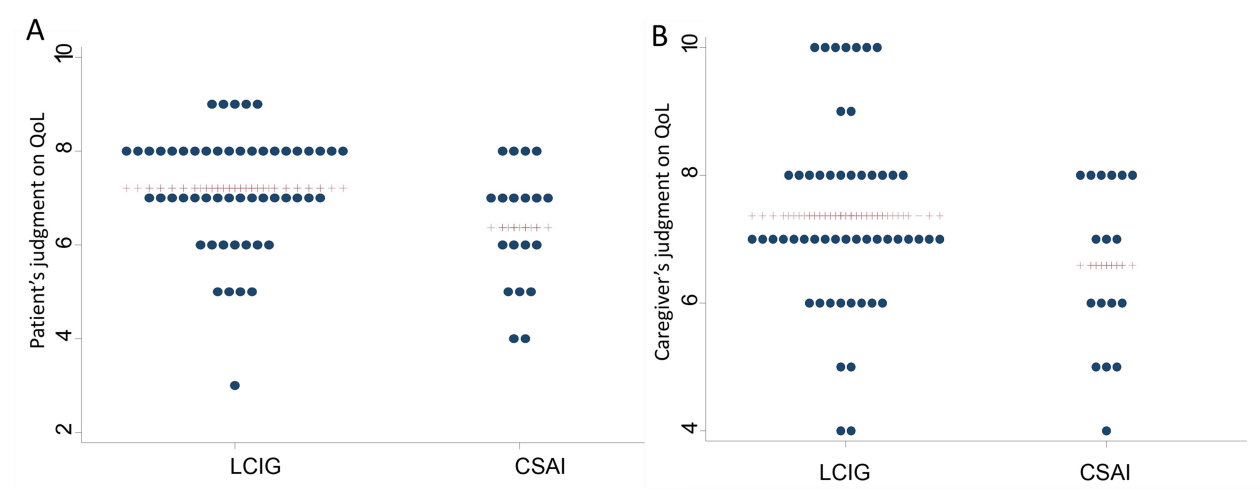

Figure 3 Perception of Quality of Life expressed by patients (A) and caregivers (B).

Note: The blue dots represent each patient score while the red crosses represent the mean value.

vitality and mental health compared to baseline, even if no significant relationship was found between these factors and the burden. ${ }^{17}$ In another study, a parallel trend between caregivers' QoL, depression, burden, sleep and patients' QoL, depression and sleep has been observed. ${ }^{28}$ We found it noteworthy in our study, that although more than $50 \%$ of the patients had sleep disturbances and approximately one third of the patients had a feeling of depressed mood, the intensity of the caregiver's burden was mild or moderate.

It has also been observed that notwithstanding a slightly higher frequency of depressive state and sleep disorders in LCIG caregivers compared to CSAI caregivers, the former tended to have a higher improvement of mood (38\% vs. 30\%) and reported a higher QoL perception ( 7.37 vs. 6.59 ) compared to the latter.

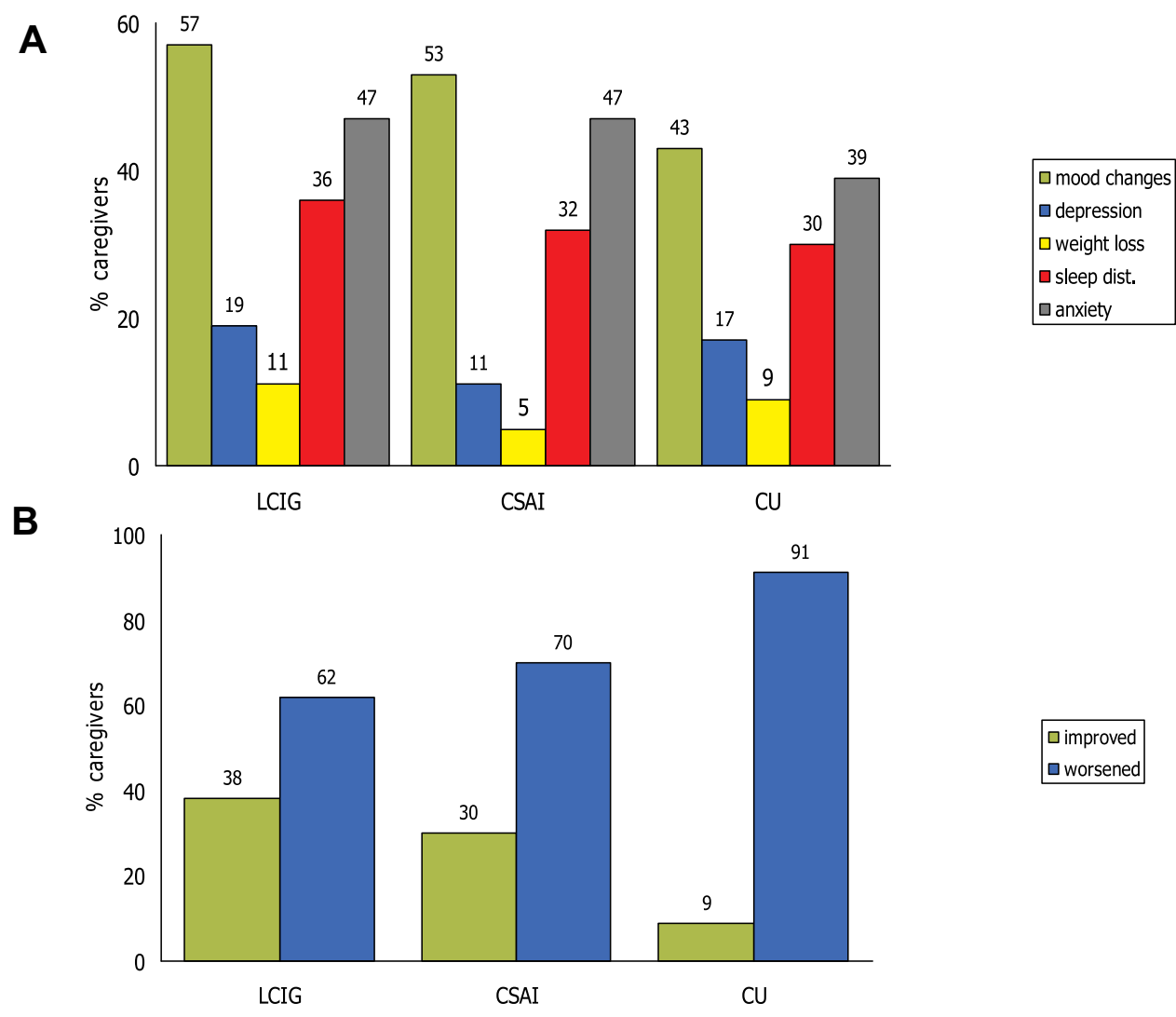

Figure 4 Box-plot of ZBI scores in each population. 
In this study, patient QoL was significantly better in the LCIG and CSAI groups compared with the CU group according to PDQ-8 scores. Moreover, as reported in the previously published data on PREDICT, both LCIG and CSAI patients had QoL that was good or very good whereas $65 \%$ of CU patients had poor or very poor QoL. It is interesting to observe that there was a moderate correlation between the daily OFF duration and the PDQ- 8 score, suggesting a possible worsening of QoL with the increasing daily OFF hours. Further data will be necessary to understand the impact of PD symptoms on patient's QoL.

In our study, the questionnaire to caregivers gave the possibility to detect some difference in the level of social adaptation among the three groups of care partners. In fact, the most satisfactory level for LCIG or CSAI caregivers was expressed for the time availability for family duties or for leisure activities, while the $\mathrm{CU}$ caregivers expressed a negative opinion. The CU caregivers' opinion is in line with the conclusion reported by Glozman et al where a correlation between the caregivers' dissatisfaction with their own QoL and the level of their input into helping the patient with everyday living was found using the SQLC. ${ }^{24}$

\section{Limitations and Strengths}

Our study further increases the knowledge on caregiver burden in advanced PD. As reported in previous trials, ${ }^{6,11}$ patient QoL is one of the main influencing factors of carers' burden and the ZBI questionnaire should be considered a valid instrument to assess the burden of PD in a unidimensional construct, even if burden has been recently considered multifactorial with personal strain and role strain as common factors indicating a complexity of its use especially in PD with dementia. ${ }^{32}$ In our study we have excluded patients with dementia and this could represent a limitation in the burden detection of this population considering that it has been reported that the presence of dementia in PD patients significantly increases caregiver burden and decreases QoL. ${ }^{33}$

The main limitation of this study is its cross-sectional design, which has not allowed consideration on the evolution of the burden of care and a comparison with baseline condition before treatment initiation. Moreover, the differences in sample size compared to CSAI group has not allowed a robust comparison with LCIG group. Furthermore, we did not consider in this study the burden of caregivers assisting patients treated with DBS. The selfassessment rating on caregivers' current QoL used in our study is not a validated scale and this represents another limitation, as well as the fact that in most of the cases, the caregivers gave their assistance to patients since more than 12 months, which could have hidden the potential benefit perception in QoL coming from the questionnaires. In any case, the combination of the assessment of the ZBI score and a very simple questionnaire that allow to understand the daily QoL of the care partners could represent an interesting way to evaluate the burden due to long-term assistance to PD patients.

\section{Conclusion}

In summary, our study on caregiver burden offers important insights into the role of care partners. In addition to daily family activities and/or working duties, caregivers have numbers of obligations, which tend to progressively increase burden and subsequently reduce their QoL.

In this further analysis we showed that despite the impact of the daily assistance to the patient, the reduction of caregiver burden was accompanied by an improvement in their QoL perception and in their ability to perform other family duties or to have more time for themselves.

Caregiver burden should be considered in the management of people affected by advanced PD in order to improve the lives of both carers and patients and to reduce possible caregivers' comorbidities and loss of socioeconomic resources.

\section{Funding}

This work was funded by AbbVie Srl. AbbVie participated in the study design, research, data collection, analysis, and interpretation of data, and writing, reviewing, and approving the publication.

\section{Disclosure}

N. Modugno reports fees for oral presentations from AbbVie, UCB, Zambon and Bial. A. Antonini has received compensation for consultancy and speaker related activities from UCB, Boehringer Ingelheim, AbbVie, Zambon, Bial, Ever Pharma, Neuroderm, Therevance, Biogen; he receives research support from Chiesi Pharmaceuticals, Lundbeck, Horizon 2020 - PD_Pal Grant 825,785, Ministry of Education University and Research (MIUR) Grant ARS01_01081. He serves as consultant for BoehringerIngelheim for legal cases on pathological gambling". A. Tessitore declares speaking honoraria and travel expenses for attending meetings from AbbVie. P. Marano declares consultancy fees from AbbVie. F.E. Pontieri received 
honoraria for speaker activity from Zambon, AbbVie, Bial, compensation for serving in the Steering Committee of an international grant from AbbVie; he also received an unconditioned grant for research activity from Zambon. N. Tambasco received speaker honoraria from Lundbeck and AbbVie. M. Canesi declares grants from UCB, Zambon and Ralpharma. G. Fabbrini received payment for International Congress from Zambon; he is also the editorial board for Parkinsonism and Related Disorders. R. Quatrale has received honoraria for consulting services and symposia from AbbVie and Zambon. P. Solla has received honoraria for participation in advisory boards from AbbVie. G. Gualberti, G. Melzi, are employees of AbbVie Italy and may own AbbVie stocks/options. The other authors report no conflicts of interest in this work.

\section{References}

1. Bhimani R. Understanding the burden on caregivers of people with Parkinson's: a scoping review of the literature. Rehabil Res Pract. 2014;718527. doi:10.1155/2014/718527

2. Mosley PE, Moodie R, Dissanayaka N. Caregiver burden in parkinson disease: a critical review of recent literature. $J$ Geriatr Psychiatry Neurol. 2017;30(5):235-252.

3. Martínez-Martín P, Forjaz MJ, Frades-Payo B, et al. Caregiver burden in parkinson's disease. Mov Dis. 2007;22(7):924-931. doi: $10.1002 / \mathrm{mds} .21355$

4. Santos-García D 1, de la Fuente-fernández R. Factors contributing to caregivers' stress and burden in parkinson's disease. Acta Neurol Scand. 2015;131(4):203-210. doi:10.1111/ane.12305

5. Schrag A, Hovris A, Moreley D, et al. Caregiver-burden in parkinson's disease is closely associated with psychiatric symptoms, falls, and disability. Parkins Rel Disord. 2006;12:35-41. doi:10.1016/j. parkreldis.2005.06.011

6. Oh YS, Lee JE, Lee PH, et al. Neuropsychiatric symptoms in parkinson's disease dementia are associated with increased caregiver burden. J Mov Disord. 2015;8:26-32. doi:10.14802/jmd.14019

7. Sensi M, Cossu G, Mancini F, et al. On behalf of the Italian Levodopa Carbidopa Intestinal Gel Working Group. Which patients discontinue? Issues on levodopa/carbidopa intestinal gel treatment: Italian multicentre survey of 905 patients with long-term follow-up. Parkins Rel Disord. 2017;38:90-92. doi:10.1016/j.parkreldis.2017.02.020

8. Greenwell K, Gray WK, van Wersch A, van Schaik P, Walker R. Predictors of the psychosocial impact of being a carer of people living with parkinson's disease: a systematic review. Parkins Rel Disord. 2015;21(1):1-11. doi:10.1016/j.parkreldis.2014.10.013

9. Rektorova I. Current treatment of behavioral and cognitive symptoms of parkinson's disease. Parkins Rel Disord. 2019;59:65-73. doi:10.1016/j.parkreldis.2019.02.042

10. Torny F, Videaud H, Chatainier P, Tarrade C, Meissner WG, Couratier P. Factors associated with spousal burden in parkinson's disease. Rev Neurol. 2018;174(10):711-715. doi:10.1016/j.neurol.2018.01.372

11. Martinez-Martin P, Rodriguez-Blazquez C, Forjaz MJ. Quality of life and burden in caregivers for patients with parkinson's disease: concepts, assessment and related factors. Expert Rev Pharmacoecon Outcomes Res. 2012;12:221-230. doi:10.1586/erp.11.106

12. Dickens CM, McGowan L, Percival C. Lack of a close confidant, but not depression, predicts further cardiac events after myocardial infarction. Heart. 2004;90(5):518-522. doi:10.1136/hrt.2003.011668
13. Benavides O, Alburquerque D, Chaná-Cuevas P. Burden among caregivers of patients with parkinson disease. Rev Med Chil. 2013;141(3):320-326. doi:10.4067/S0034-98872013000300006

14. Lökk J. Caregiver strain in parkinson's disease and the impact of disease duration. Eur J Phys Rehabil Med. 2008;44(1):39-45.

15. Antonini A, Nitu B. Apomorphine and levodopa infusion for motor fluctuations and dyskinesia in advanced parkinson disease. $J$ Neural Transm. 2018;125(8):1131-1135. doi:10.1007/s00702-018-1906-0

16. Lewis CJ, Maier F, Horstkötter N, et al. The impact of subthalamic deep brain stimulation on caregivers of parkinson's disease patients: an exploratory study. $J$ Neurol. 2015;262(2):337-345. doi:10.1007/ s00415-014-7571-9

17. Ciurleo R, Corallo F, Bonanno L, et al. Assessment of duodopa ${ }^{\circledR}$ effects on quality of life of patients with advanced parkinson's disease and their caregivers. $J$ Neurol. 2018;265(9):2005-2014. doi:10.1007/s00415-018-8951-3

18. Tessitore A, Marano P, Modugno N, et al. Caregiver burden and its related factors in advanced parkinson's disease: data from the PREDICT study. J Neurol. 2018;265(5):1124-1137. doi:10.1007/ s00415-018-8816-9

19. Mosley PE, Breakspear M, Coyne T, Silburn P, Smith D. Caregiver burden and caregiver appraisal of psychiatric symptoms are not modulated by subthalamic deep brain stimulation for parkinson's disease. NPJ Parkinsons Dis. 2018;4:12. doi:10.1038/s41531-0180048-2

20. Soileau MJ, Persad C, Taylor J, Patil PG, Chou KL. Caregiver burden in patients with parkinson disease undergoing deep brain stimulation: an exploratory analysis. J Parkinsons Dis. 2014;4(3):517-521. doi:10.3233/JPD-140380

21. Crespo-Burillo JA, Rivero-Celada D, Saenz-de Cabezón A, CasadoPellejero J, Alberdi-Viñas J, Alarcia-Alejos R. Deep brain stimulation for patients with parkinson's disease: effect on caregiver burden. Neurologia. 2018;33(3):154-159. doi:10.1016/j.nrl.2016.05.017

22. Chattat R, Cortesi V, Izzicupo F, et al. The Italian version of the zarit burden interview: a validation study. Int Psychogeriatrics. 2011;23:797-805. doi:10.1017/S1041610210002218

23. Peto V, Jenksinson C, Fitzpatrick R. PDQ-39: a review of the development, validation and application of a parkinson's disease quality of life questionnaire and its associated measures. J Neurol. 1998;245:S10-S14.

24. Glozman JM, Bicheva KG, Fedorova NV. Scale of Quality of Life of Care-Givers (SQLC). J Neurol. 1998;245(Suppl S1):S39-S41. doi:10.1007/PL00007738

25. Martinez M, Multani N, Anor CJ, et al. Emotion detection deficits and decreased empathy in patients with alzheimer's disease and parkinson's disease affect caregiver mood and burden. Front Aging Neurosci. 2018;10:120. doi:10.3389/fnagi.2018.00120

26. Martinez-Martin P, Rodriguez-Blazquez C, Forjaz MJ, et al. Neuropsychiatric symptoms and caregiver's burden in Parkinson's disease. Parkins Rel Disord. 2015;21(6):629-634. doi:10.1016/j. parkreldis.2015.03.024

27. Grün D, Pieri V, Vaillant M, Diederich NJ. Contributory factors to caregiver burden in parkinson disease. J Am Med Dir Assoc. 2016;17 (7):626-632. doi:10.1016/j.jamda.2016.03.004

28. Bartolomei L, Pastore A, Meligrana L, et al. Relevance of sleep quality on caregiver burden in parkinson's disease. Neurol Sci. 2018;39(5):835-839.

29. Lau KM, Au A. Correlates of informal caregiver distress in parkinson's disease: a meta-analysis. Clin Gerontol. 2011;34:117-131. doi:10.1080/07317115.2011.539521

30. Pinquart M, Sörensen S. Associations of stressors and uplifts of caregiving with caregiver burden and depressive mood: a meta-analysis. J Gerontol B Psychol Sci SocSci. 2003;58(2):P112-P128.

31. Ozdilek B, Gunal DI. Motor and non-motor symptoms in Turkish patients with parkinson's disease affecting family caregiver burden and quality of life. $J$ Neuropsychiatry Clin Neurosci. 2012;24:478-483. doi:10.1176/appi.neuropsych.11100315 
32. Vatter S, McDonald KR, Stanmore E, Clare L, Leroi I. Multidimensional care burden in parkinson-related dementia. J Geriatr Psychiatry Neurol. 2018;31(6):319-328. doi:10.1177/ 0891988718802104

33. Leroi I, McDonald K, Pantula H, Harbishettar V. Cognitive impairment in parkinson disease: impact on quality of life, disability, and caregiver burden. J Geriatr Psychiatry Neurol. 2012;25(4):208-214. doi:10.1177/0891988712464823
34. Zarit SH, Zarit JM; 1983. Available from: www.mapi-trust.org, https:/eprovide.mapi-trust.org/. Accessed November 25, 2020.

\section{Publish your work in this journal}

Neuropsychiatric Disease and Treatment is an international, peerreviewed journal of clinical therapeutics and pharmacology focusing on concise rapid reporting of clinical or pre-clinical studies on a range of neuropsychiatric and neurological disorders. This journal is indexed on PubMed Central, the 'PsycINFO' database and CAS, and is the official journal of The International Neuropsychiatric Association (INA). The manuscript management system is completely online and includes a very quick and fair peer-review system, which is all easy to use. Visit http://www.dovepress.com/testimonials.php to read real quotes from published authors. 